\title{
Aplicación de la metodología de la gestión BIM en el canal de conducción de agua del sistema de riego Chiticay - Paute
}

Application of the BIM management methodology in the water conduction channel of the Chiticay - Paute irrigation system

Chica Guzmán Carlos Adrián. ${ }^{1}$ \& Diego Fernando Coronel Sacoto. ${ }^{2}$

Recibido: 04-05-2021 / Revisado: 15-05-2021 /Aceptado: 08-06-2021/ Publicado: 05-07-2021

\begin{abstract}
.
DOI: https://doi.org/10.33262/concienciadigital.v4i3.1761

Introduction: This research investigates the most relevant programs used in the BIM management methodology worldwide to manage civil projects in terms of linear infrastructures, this information allowed to propose the implementation of this methodology in the management of the irrigation system Chicticay-Paute to optimize the cost of the work with the efficient use of material and human resources. Objective. Obtain information from bibliographic sources and experts distributed in Europe and America on the tools used for BIM management in civil construction processes focused on drinking water networks. Methodology. It began by conducting a survey of different BIM, hydraulic and road specialists who are involved in this type of management from different countries in Europe and America to verify which are the most accessible tools and which gives the best results for the development of the third, fourth and fifth dimension of project management. In addition, a bibliographic review is carried out on investigations that implement the BIM methodology to complement the data presented by the experts and verify the level of maturity in the use of these tools in different countries. In addition, a section of the canal was modeled and the exact items were quantified to optimize the
\end{abstract}

\footnotetext{
${ }^{1}$ Universidad Católica de Cuenca, Facultad de Arquitectura. Cuenca, Ecuador, carlos.chica1@est.ucacue.edu.ec ORCID https://orcid.org/0000-0001-8758-4069

${ }^{2}$ Universidad Católica de Cuenca, Facultad de Arquitectura. Cuenca, Ecuador, dcoronels@ucacue.edu.ec, ORCID https://orcid.org/0000-0001-8105-4104
} 
resources and time of the aforementioned irrigation system. Results. From the data obtained by the research tools and the bibliographic review, it was found that BIM technology reduced the costs of executing works for the reconstruction of the canal by 13.08 dollars in the 20 meters analyzed, while, in the $17 \mathrm{~km}$ that owns the total dimension of the channel is calculated a saving of \$11,118.00 dollars. In addition, time is optimized in the design and construction in the change of the hydraulic section of the channel. Conclution. It is concluded that the BIM methodology is suitable to be applied in several irrigation systems in the province of Azuay to save resources and interconnect several management processes in a single collaborative system in real time.

Keywords: Irrigation, BIM methodology, construction, design, resources, time.

\section{Resumen.}

Introducción: La presente investigación, indaga en los programas más relevantes utilizados en la metodología de la gestión BIM a nivel mundial para gestionar los proyectos civiles en cuanto a infraestructuras lineales, esta información permitió proponer la implementación de esta metodología en la conducción del sistema de riego ChicticayPaute para optimizar el costo de la obra con el uso eficiente de los recursos materiales y humanos. Objetivo. Obtener información de fuentes bibliográficas y de expertos distribuidos en Europa y América sobre las herramientas utilizadas para la gestión BIM en procesos de construcciones civiles de obra lineal como canales de riego. Metodología. Se inició realizando una encuesta a diferentes especialistas BIM, hidráulicos y viales que están involucrados en este tipo de gestión de diferentes países de Europa y América para constatar cuales son las herramientas más accesibles y que da los mejores resultados para el desarrollo de la tercera, cuarta y quinta dimensión de la gestión de proyectos. En adición, se realiza una revisión bibliográfica sobre investigaciones que implementan la metodología BIM para complementar los datos presentados por los expertos y verificar el nivel de madures del uso de estas herramientas en diferentes países. Además, se efectuó el modelado de un tramo del canal y se cuantificó los rubros exactos para optimizar los recursos y tiempo del sistema de riego antes mencionado. Resultados. De los datos obtenidos por las herramientas de investigación y la revisión bibliográfica se pudo constatar que la tecnología BIM redujo los costos de ejecución de obra para la reconstrucción del canal en 13,08 dólares en los 20 metros analizados, mientras que, en los $17 \mathrm{~km}$ que posee la dimensión total del canal se calcula un ahorro de \$11.118,00 dólares. Además, se optimiza el tiempo en el diseño y construcción en el cambio de la sección hidráulica del canal. Conclusión. Se concluye que la metodología BIM es apto para ser aplicado en varios sistemas de riego de la provincia del Azuay para ahorrar recursos e interconectar varios procesos de la gestión en un solo sistema colaborativo en tiempo real

Palabras claves: Riego, metodología BIM, construcción, diseño, recursos, tiempo. 


\section{Introducción.}

La preservación y uso eficiente de los recursos hídricos junto a los servicios y productos derivados de estos, son necesarios para asegurar la sostenibilidad global y la calidad de vida de las personas, sin embargo, la modernidad, el crecimiento poblacional y económico han impulsado una creciente demanda de estos recursos que representan un verdadero reto que la humanidad debe superar si quiere asegurar la subsistencia de generaciones futuras (The United Nations World Water Development Report [WWDR], 2019). Como mencionan los autores Pérez, Leyva, \& Gómez (2018), la reducción de agua disponible es un evento inminente que amenaza la estabilidad y seguridad internacional puesto que, según estimaciones, para el año 2050 la población incrementará a 9.1 mil millones de personas, esto ocasionará una demanda de alimentos y servicios hídricos sin precedentes para el planeta especialmente para el sector agrícola puesto que, como menciona el Banco Mundial (2017) se destina hasta el 70\% del agua para riego y fines agrarios a través de sistemas de distribución con índices bajos de eficiencia.

Por lo expuesto, pese a que el riego representa un porcentaje mayoritario del uso de recursos hídricos en el mundo, existen muchas zonas de la población que se dedica a la producción agrícola que aún no utiliza de forma eficiente este recurso, especialmente en los sistemas de distribución, puesto que, como mencionan Nieto, Pazmiño, Rosero, \& Quishpe (2018): "La deficiencia en el manejo del agua de riego se observa en todos los componentes del sistema, desde la captación hasta la aplicación en las parcelas; pasando por la conducción, almacenamiento, distribución y métodos de riego parcelario" (p.52).

Seguidamente, en los últimos años, se han desarrollado estrategias y sistemas de distribución de agua potable y de riego que permiten que el recurso acuífero sea abastecido de forma más eficiente en factores de cantidad, continuidad y calidad. Sin embargo, para que estos sistemas funcionen adecuadamente, es necesario que se ejecuten las etapas de construcción y mantenimiento de forma coordinada, puesto que, si existe una mala distribución y uso deficiente de los recursos tanto humanos como materiales durante la implementación de los sistemas de distribución, pueden ocasionar que los sistemas operen con caudales mucho mayores para los que fueron diseñados, en consecuencia, la eficiencia de los mismos se ve comprometido (Gómez, Cubillo, \& Carrasco, 2017).

Por lo anterior mencionado, es necesario implementar estrategias de organización y trabajo colaborativo en la construcción de estos sistemas para gestionar de forma eficiente los recursos disponibles. Una de las metodologías que cobró relevancia en los últimos años desde sus primeros conceptos en la década de los 70, es la tecnología BIM por sus siglas en inglés Building Information Modeling o también llamado construcción inteligente que integra las herramientas digitales para gestionar la construcción de una obra en tiempo real en un único modelo que constituye los datos de todos los participantes del proyecto para determinar requerimientos de compras, tiempo, impacto ambiental, mantenimiento entre otros. El autor Bermejo y González (2018) define a la metodología BIM como: 
Una representación digital de las características físicas y funcionales de una instalación. BIM es un recurso de conocimiento compartido para obtener información sobre una instalación que constituye una base fiable para las decisiones durante su ciclo de vida; definido como válido desde la concepción inicial hasta la demolición. (p.9)

En adición, esta metodología permite la verificación de manera práctica de los beneficios intangibles de un proyecto como; la comunicación multidimensional efectiva, el flujo de información, la fácil determinación de mejoras en soluciones constructivas y una toma de decisiones oportunas sin improvisación. Además, este modelo de gestión permite analizar todas las dimensiones de una obra, incluido el aspecto económico y la detección de problemas durante la construcción con sistemas complejos (Montoya, Garrigos, Echarri, \& Rizo., 2020).

A lo mencionado, para la aplicación de la metodología BIM en infraestructuras lineales como los canales en proyectos hidráulicos, es necesario la consideración de las dimensiones que posee este modelo de gestión, para verificar en qué grado de madurez se encuentra y las herramientas disponibles para cada dimensión. A continuación, se describen brevemente cada una de estas dimensiones del BIM que se relacionarían con la investigación.

La primera dimensión hace referencia al carácter unidimensional (BIM 1D) de la metodología y hace referencia a la conceptualización del proyecto de construcción, es decir, es el proceso de estudios preliminares requeridos para determinar la viabilidad, o en otras palabras, es la evaluación del grupo de expertos para darle su forma inicial y estimada (Olawumi \& Chan, 2019). La segunda dimensión se refiere al enfoque bidimensional (BIM 2D), esta dimensión es considerado como los documentos constructivos básicos para el inicio del proyecto, antes de la innovación del BIM. Son utilizados como modelos o guías para el desarrollo tridimensional (Acampa, 2019).

A lo expuesto, la tercera dimensión hace mención al enfoque tridimensional (BIM 3D) que es la representación de un modelo virtual tridimensional, donde no solo se pueden evidenciar líneas o representaciones, sino propiedades ligadas a las herramientas del diseño. Según el programa utilizado podrá ser exportado a un programa con un archivo común. Por otro lado, la cuarta dimensión se refiere a la programación temporal (BIM 4D) que, en palabras de los autores Bermejo \& González (2018):

Es el resultado de añadir la variable tiempo al modelo BIM 3D. Permite controlar la dinámica del proyecto, realizar simulaciones de las diferentes fases de la ejecución de la obra o diseñar el plan de ejecución y compartirlo con todos los oficios, empresas y demás agentes intervinientes en el proceso (...) Además, esta dimensión permite simular el proceso de ejecución, visualizando el avance de la obra, el equipamiento y medios auxiliares que se utilizarán, su correcto emplazamiento, las interferencias que se puedan llegar a producir o las medidas de seguridad y salud que será necesario utilizar para evitar accidentes. (p.24) 
Finalmente, la quinta dimensión (BIM 5D) Es la gestión de los costos, la cual está vinculada de una manera óptima cuando se logra un modelado eficiente y completo del proyecto. En esta dimensión ya se posee la planificación detallada de las actividades a ejecutarse, vinculadas directamente con los rendimientos u otros parámetros para la estimación de costos. Los softwares utilizados permitirán la generación de un presupuesto detallado en tiempo real (Vera, 2018).

A todo lo anterior expuesto, estas herramientas se pueden utilizar para gestión de forma eficiente en la construcción de la línea de conducción de agua destinada para riego en las cinco dimensiones, de esta manera, se puede evitar los problemas recurrentes de su construcción y posterior mantenimiento que infieren en la disminución de eficiencia, disponibilidad, y costos elevados, especialmente en la realidad ecuatoriana. Puesto que, en Ecuador, el mantenimiento de la line de conducción de agua en muchos de los casos, especialmente en la zona andina, se realiza a través de grupos de beneficiarios del recurso que organizan mingas (reunión de tipo comunitaria social para realizar trabajos) de mantenimiento (El Comercio, 2014). Pero, esto no siempre se cumple, ya que se realizan estas acciones de manera poco oportuna y de forma inadecuada, con soluciones poco duraderas que solo alivian los problemas.

La organización insipiente, desordenada y poco eficiente del sistema de trabajo anteriormente mencionado, repercute directamente en la vida útil de los sistemas, puesto que permite la acumulación de sedimentos, desgaste del recubrimiento, fugas en tramos importantes e interrupciones del servicio que provoca un desperdicio del recurso hídrico y de los recursos económicos de la zona afectada (Goulven, Ribadeneira, \& Ruf., 1991). Como consecuencia de estos problemas se han creado Instituciones que manejan el recurso agua de acuerdo a sus competencias como los Gobiernos Provinciales que son los encargados del diseño, construcción y mantenimiento de los sistemas de riego a nivel provincial. Sin embargo, los sistemas de riego no han coordinado de una manera apropiada las etapas de construcción y mantenimiento, afectando en gran magnitud a los usuarios de dichos sistemas.

En este sentido, el sistema de riego Chicticay- Paute, ubicado en el cantón Paute perteneciente a la provincia del Azuay, es uno de los sistemas más afectados por las circunstancias mencionas, debido a que, pese a ser uno de los sistemas con mejor operatividad a nivel del austro ecuatoriano, la sección hidráulica está sobredimensionada y provoca un mantenimiento anual innecesario por su diseño inadecuado. Este sistema entró en servicio en la década de los 80, el cual comprende de una conducción de 17 km y posee un caudal autorizado de 300 1/s, el área de riego del sistema es de 1000 ha. Debido a que ha cumplido su vida útil, se hace imprescindible su rehabilitación emergente, ya que presenta problemas de filtraciones en la red de conducción a cielo abierto provocando deslizamientos de pequeñas a grandes dimensiones de los taludes permanentemente.

Por las razones anteriormente mencionadas, el presente artículo pretende implementar la metodología de la gestión BIM en el sistema de riego Chiticay - Paute para buscar eficiencia hidráulica y optimizar los recursos humanos y materiales del tramo restaurado, 
como muestra, para luego ser aplicado a toda la extensión del canal. Para realizar lo anterior mencionado se procede a evaluar el estado general del revestimiento de hormigón que povocan las filtraciones y en consecuencia los deslizamientos. Además, se indaga en cómo se aplica la gestión BIM mediante softwares existentes en el medio profesional a través de una recopilación de información de expertos a nivel mundial y con la recopilación bibliográfica de investigaciones que abordan esta temática.

\section{Metodologia.}

Esta investigación se basa en la metodología presentada por Cabré (2012) que corresponde al tipo cuasi - experimental que tiene las siguientes características de acuerdo a distintos autores. (ver Tabla 1)

Tabla 1. Características de la investigación cuasi-experimental

\begin{tabular}{ll} 
Autor & Característica \\
\hline $\begin{array}{l}\text { Cook y } \\
\text { Campbell }\end{array}$ & $\begin{array}{l}\text { Se diferencia de la investigación experimental por la ausencia de } \\
\text { asignación aleatoria a las variables, es decir, el investigador no tiene } \\
\text { control de las unidades investigadas. }\end{array}$ \\
$\begin{array}{l}\text { Pedhazur } \\
\text { y }\end{array}$ & $\begin{array}{l}\text { El cuasi-experimento posee todos los elementos constitutivos de una } \\
\text { experimento pero, el investigador no asigna aleatoriamente los grupos de } \\
\text { investigación, en consecuencia, se ve obligado a evaluar y separar los } \\
\text { factores que afectan la variable dependiente }\end{array}$ \\
Hedrick & $\begin{array}{l}\text { "Permiten estimar los impactos del tratamiento o programa, dependiendo } \\
\text { de si llega a establecer una base de comparación apropiada". (p.58) }\end{array}$
\end{tabular}

De acuerdo a la información presentada en la tabla anterior y complementado con el análisis del diseño cuasi experimental presentado por el Centro de Estudios de Opinión [CEO] (2008), esta investigación se abordó por etapas que se describirán a continuación: En la primera etapa se realizó una exploración bibliográfica practica para indagar en investigaciones que abordan las cinco dimensiones de la gestión de proyectos a través de las herramientas virtuales de la metodología BIM con el propósito de caracterizar el nivel de madurez de esta metodología en infraestructuras lineales en el campo de la ingeniería civil, y obtener instrumentos digitales que se puedan implementar en la gestión del tramo Chiticay-Paute.

Para la segunda etapa, se elaboró un cuestionario de tipo exploratorio para indagar el nivel de madurez y las herramientas que se usan actualmente en el campo profesional de la ingeniería civil en cuanto a la gestión de proyectos, mediante la aplicación de la metodología BIM y sus herramientas virtuales. Para esto, se aplicaron las encuestas a diferentes expertos en la metodología BIM en varios países de Europa y América.

En la tercera etapa se continuó con el análisis y evaluación del tramo del canal ChiticayPaute a través de sus 4 dimensiones. En la primera dimensión, se realiza un levantamiento 
del tramo de la línea de conducción a través de la herramienta de "estación total" que utiliza puntos de referencia para verificar su longitud total, a través de esta herramienta se determinó la dimensión de 19 metros. A continuación, se procedió a medir la sección del canal mediante el uso de un flexómetro y se verifico que el canal posee una sección variable entre $80 \mathrm{~cm}$ x $80 \mathrm{~cm}$ a $90 \mathrm{~cm}$ x $90 \mathrm{~cm}$.

Para la segunda dimensión, que hace referencia al diseño de la nueva sección de la línea de conducción, se utilizó el programa HydraFlow en la cual se colocan parámetros de caudal, pendiente, coeficiente de rugosidad del material, y la base variable para determinar la sección optima del canal. Además, se implementó las herramientas encontradas en la recopilación bibliográfica y los datos de las encuestas a los expertos que corresponde al software Civilcad $3 \mathrm{~d}$ y al plug-in de autodesk-Subassambly Composer.

Con los instrumentos mencionados se realizó el dimensionamiento de la sección del canal y se determinaron las herramientas a emplearse de manera dinámica entre los programas ya que el plug-in mencionado permite que los cambios realizados en Subassambly Composer se actualice automáticamente en Civilcad. La sección diseñada se muestra en la Figura 1.

Figura 1. Sección del canal optimizado

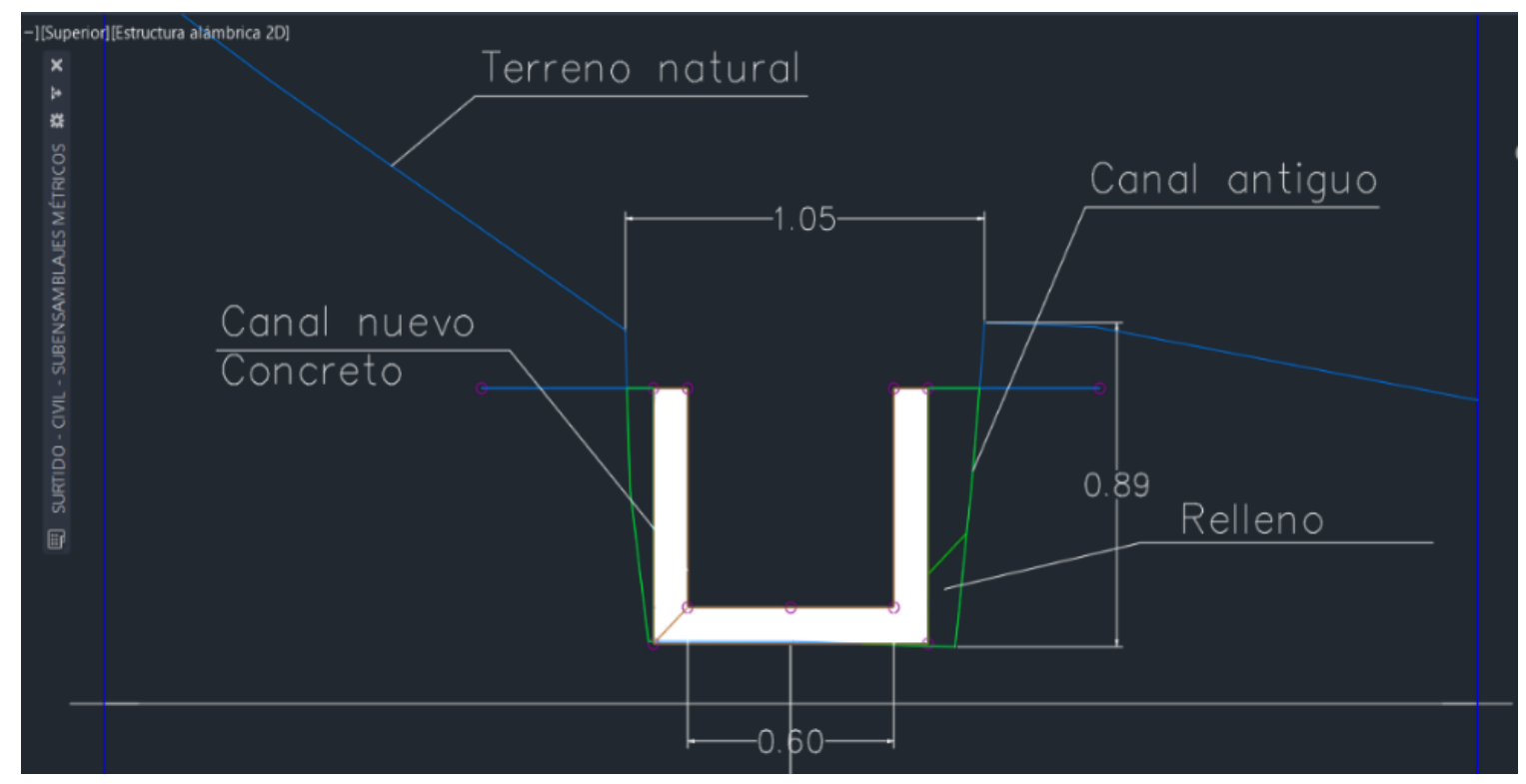

Los parámetros para el dimensionamiento de la sección hidráulica se determinaron según lo establecido por Ministerio del Ambiente y del Agua (MAE) que autorizo un caudal promedio de 300 litros/segundo para el sistema de riego Chiticay-Paute, con una pendiente del 2 por mil, además, se utilizó el valor de la rugosidad de $0.014 \mathrm{~mm}$ que corresponde al acabado superficial de los conductos hidráulicos del concreto sin pulir referenciado por Chow (2004). La codificación del canal en el programa Subassambly Composer se muestra en la figura 2. 
Figura 2. Codificación del canal en Subassambly Composer

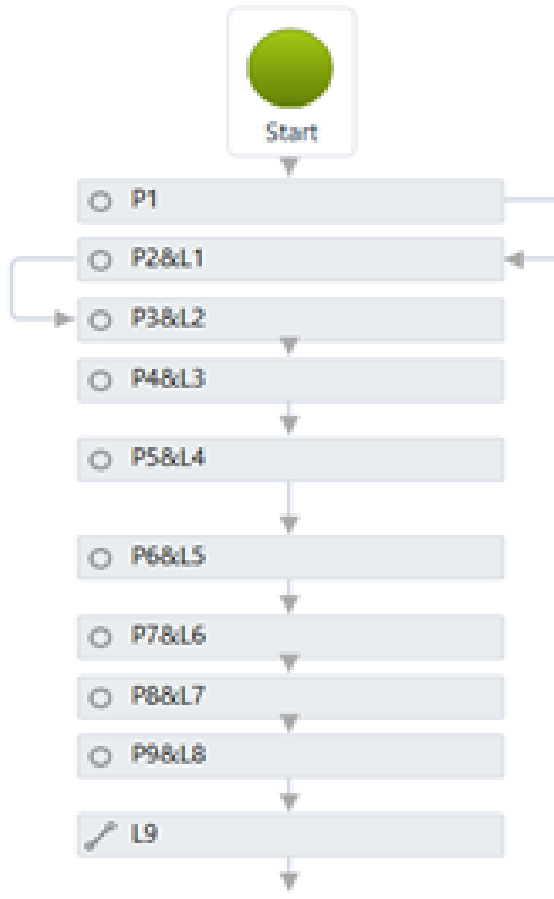

(a)

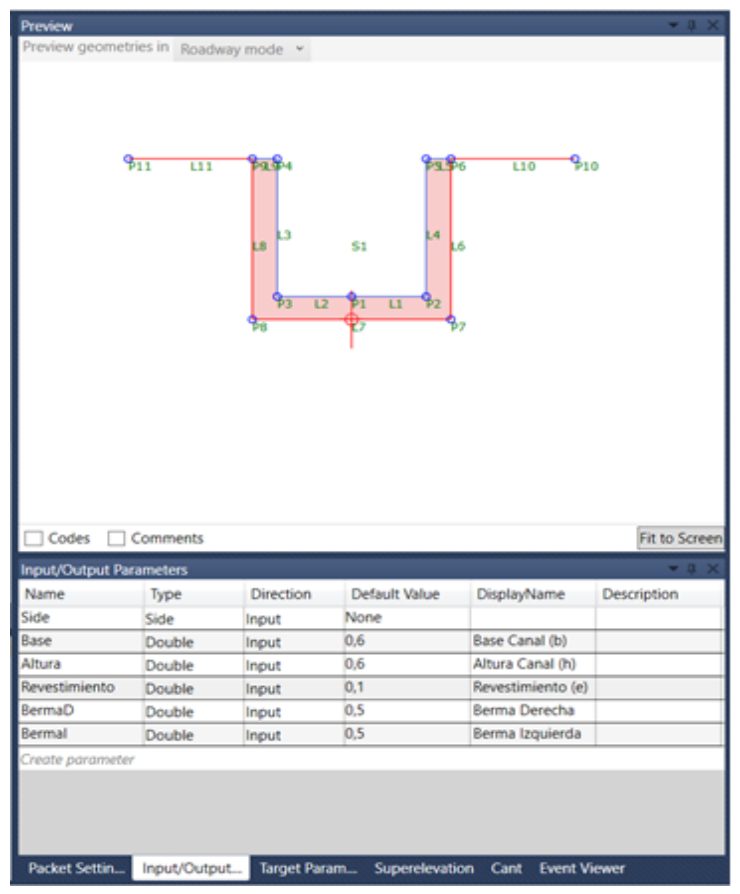

(b)

Nota: Diagrama de flujo con la codificación del diseño del canal para obtener el subensamblaje (a) Dimensiones del canal (b) Fuente: Autor (2021)

Como se muestra en la figura 3, para la tercera dimensión se realizó el diseño tridimensional del canal con los datos tomados en la primera dimensión en el programa Civil Cad 3D, este modelado se alineó al diseño de los demás programas, es decir, con el diseño hidráulico de Hydraflow que sirve para la codificación del mismo en el plug-in Subassambly Composer y el perfil del canal efectuado en el civil Cad 3D para generar el corredor del canal. Con el corredor, se realiza las superficies de obra lineal, lo que permitió generar líneas de muestreo que proporcionó las cantidades de obra óptimas para la ejecución del proyecto.

Figura 3. Líneas de muestreo del canal en Civilcad 3D.

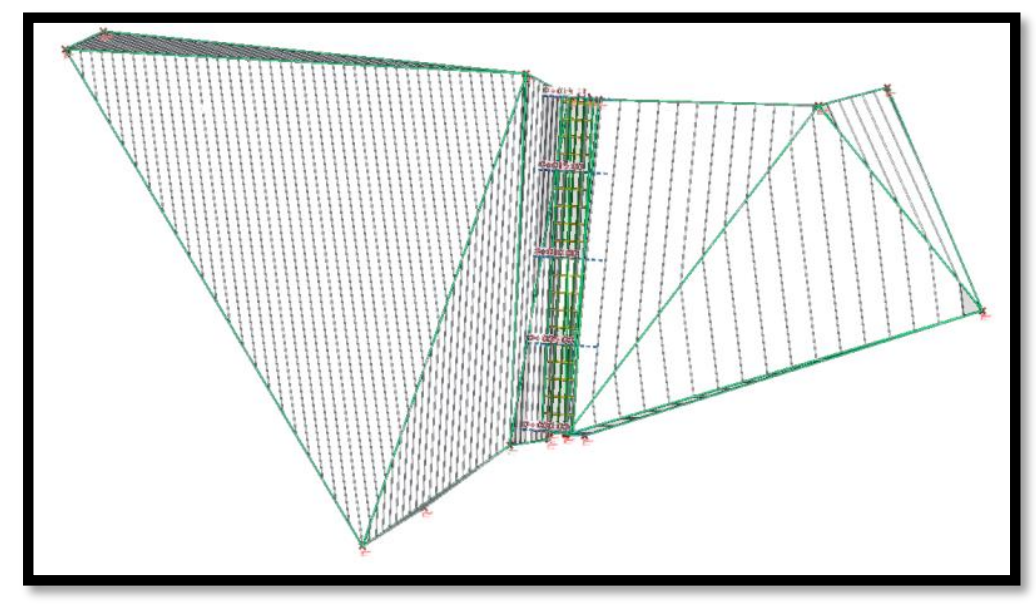


En la cuarta dimensión que corresponde a la programación temporal del 3D se utilizó los programas mencionados por los encuestados que fueron; Navisworks y Microsoft Project. Con la ayuda del programa Microsoft Project se realizó la planificación del proyecto según los componentes a emplearse, pero como el análisis está enfocado a un solo tramo, se planificó el proyecto de manera que se pueda finalizar en tres días. Para la interoperabilidad entre los dos programas, es necesario que, en programa de Microsoft Project, se incluya la columna de Navisworks Type donde se va a agregar el recurso a cuál pertenece y el identificador ID. Adicionalmente, el archivo debe guardarse en formato CSV para asegurar la compatibilidad y colocar los datos a exportar como se muestra en la figura 4.

Figura 4. Parámetros de exportación en el programa Microsoft Project

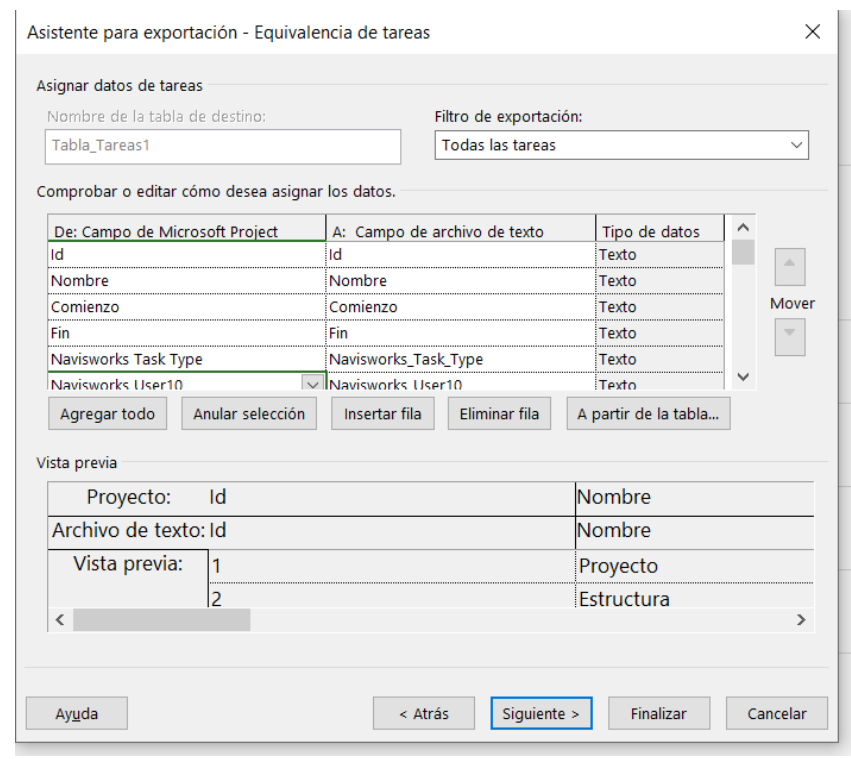

Luego de la exportación, ya se pudo dar inicio al seguimiento del proyecto a través del programa Navisworks que, a su vez, permite verificar lo diseñado en el programa CivilCAD 3D y la planificación realizada en Microsoft Poject. La forma de asegurar la interoperabilidad es la siguiente:

- En el programa de civilcad 3D cuando ya se finaliza el diseño, se debe extraer el sólido del proyecto, en este caso, el diseño del canal y guardar con formato DWG.

- En el programa NAVISWORKS se añade el proyecto guardado en civilcad $3 \mathrm{D}$

- Con el proyecto agregado en el programa, se realiza la importación de los datos del programa Microsoft Project, en este paso se puede agregar en formato CSV o en formato Microsoft Project.

\section{Resultados.}

La presentación de los resultados se divide por los objetivos planteados en esta investigación y en correspondencia las 4 dimensiones del tramo del canal Chiticay - 
Paute. Por lo mencionado, el primer resultado se corresponde al nivel de madurez en que se encuentra la gestión BIM en los proyectos civiles. Como se puede apreciar en la figura 5, para dar un mayor alcance a esta investigación se enfocó la encuesta a varios expertos alrededor del mundo.

Figura 5. País de residencia de los encuestados.

\section{¿EN QUE PAIS RESIDE?}

14 respuestas

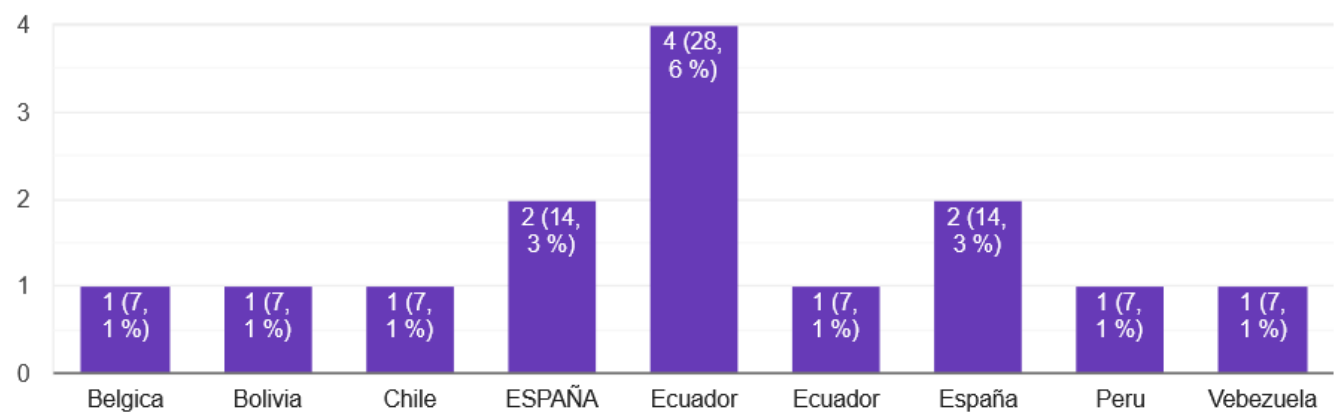

Como se puede observar, se recolectó información de expertos en gestión BIM de 9 países distintos distribuidos entre Latinoamérica y Europa, pero la población mayoritaria se encuentra en Ecuador, puesto que es el lugar en la que se desarrolla el proyecto. A esto, en la figura 5 se muestra los valores promediados del nivel de desarrollo de la metodología BIM en cada país.

Figura 5. Nivel de desarrollo de la tecnología BIM en el país de los encuestados.

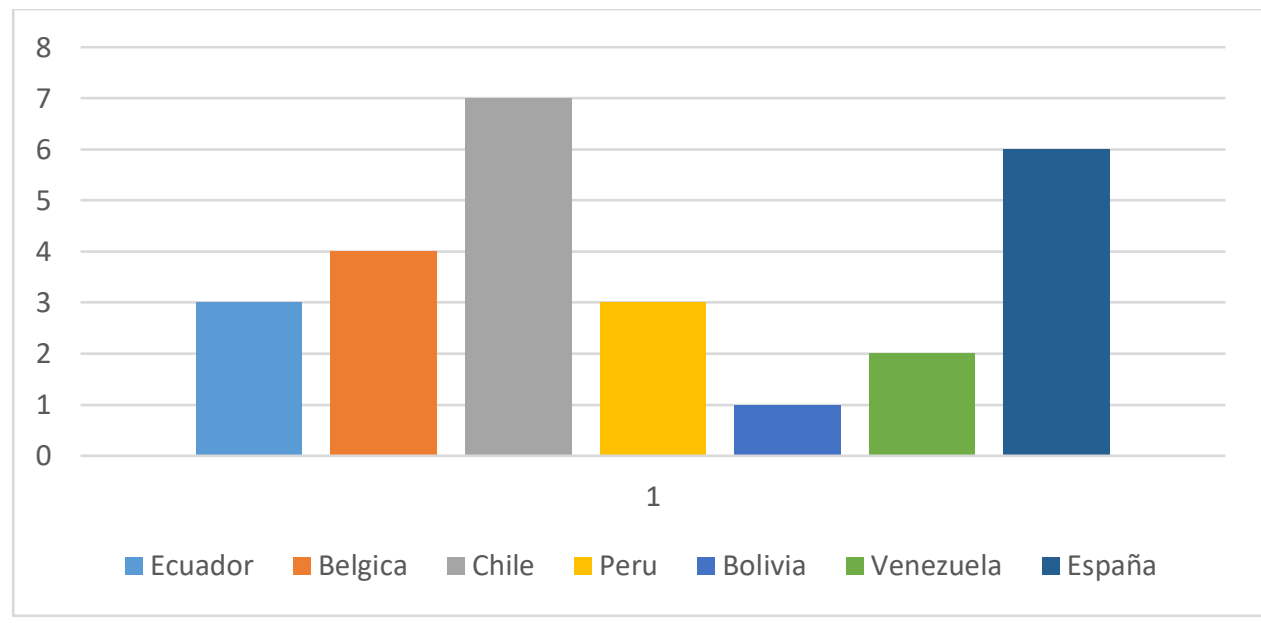

Como se puede observar en la figura anterior, conforme a las respuestas de los encuestados, los países con el mayor nivel de desarrollo de la tecnología BIM es Chile y España, mientras que el país el menor nivel es Bolivia. En cuanto a Ecuador, el promedio de respuestas es de 3 puntos que corresponde a un nivel por debajo del promedio general de todos países consultados. A continuación, en la tabla 2, se muestra los resultados de la encuesta sobre cuantas dimensiones es posible trabajar usando la tecnología BIM. 
Tabla 2. Dimensiones que han utilizado los encuestados en la tecnología BIM

\begin{tabular}{cc}
\hline País & Dimensiones \\
\hline Ecuador & $3 \mathrm{D}, 4 \mathrm{D}, 5 \mathrm{D}$. \\
Bélgica & $7 \mathrm{D}$ \\
Chile & $10 \mathrm{D}$ \\
Perú & $7 \mathrm{D}$ \\
Venezuela & Aún no han implementado \\
España & $5 \mathrm{D}$ \\
\hline
\end{tabular}

Como se puede apreciar en la tabla anterior, la metodología BIM se puede implementar hasta las 10 dimensiones como menciona el consultor de Chile, pese a esto, es importante recalcar que las 10 dimensiones solo se pueden aplicar en arquitectura pues, según la indagación bibliográfica, solo se puede llegar hasta la cuarta o quinta dimensión. A lo expuesto, en la tabla 3 se presenta las herramientas usadas por los expertos para la gestión BIM de sus proyectos.

Tabla 3. Herramientas usadas por los expertos en la gestión BIM por país.

\begin{tabular}{|c|c|c|c|}
\hline País & $\begin{array}{l}\text { Herramientas para } \\
\text { elementos hidráulicos }\end{array}$ & BIM 4D & BIM 5D \\
\hline Ecuador & $\begin{array}{l}\text { CYPE, Civil CAD 3D, } \\
\text { Revit, Cypecad. }\end{array}$ & $\begin{array}{l}\text { Revit, ArchiCAD, } \\
\text { Naviswork, PMI. }\end{array}$ & $\begin{array}{l}\text { Revit, ArchiCAD, } \\
\text { Presto, Arquímedes, } \\
\text { Cype }\end{array}$ \\
\hline Bélgica & Revit, Cypecad. & Revit, & - \\
\hline Chile & Revit, Tekla Structures & $\begin{array}{l}\text { Naviswork, Synchro, } \\
\text { Bexel Manager }\end{array}$ & - \\
\hline Perú & Revit & Naviswork & Presto con plugin Cost It \\
\hline Bolivia & - & - & - \\
\hline Venezuela & Revit & Naviswork & Maprex datalaing \\
\hline España & Civil CAD 3D, Revit, & $\begin{array}{l}\text { Synchro, Microsoft } \\
\text { Project, Presto de RIB } \\
\text { Spain }\end{array}$ & $\begin{array}{l}\text { Presto, Cost it, } \\
\text { Arquímedes }\end{array}$ \\
\hline
\end{tabular}

Para complementar la tabla anterior se muestra en la tabla 4 los instrumentos indagados en la revisión bibliográfica

Tabla 4. Instrumentos de gestión BIM encontrados en la revisión bibliográfica

\begin{tabular}{ccc} 
Autor/es & Dimensión & Instrumentos encontrados \\
\hline Vera (2018) & BIM 3D & Civil CAD3D \\
& BIM 4D & $\begin{array}{c}\text { Ms Project, Primavera para la } \\
\text { simulación del software programas } \\
\text { Synchro PRO }\end{array}$ \\
& BIM 5D & Revit, plug-in cost it, presto. \\
\hline
\end{tabular}


BIM 3D

D'Amico, D'Ascanio, \& Chiarra. (2020)

BIM 4D

BIM 5D

(Li\&Zhang\&Mei\&Lin\&Yu\&Qiu\&S u\&Lin\&Lou, 2020)

(Monar, 2020)
BIM 3D

BIM 5D BIM 2D
Civil CAD 3D

STR, Vision, CPM, Ms Project, para la 17 utodesk17n del software programas Navisworks, Synchro

PRO

5D: investigación-STR Vision CPM

Revit,

Software Glodon. Subassembly Composer, Autodesk

Como se puede observar en las tablas anteriores, los resultados tanto de la investigación bibliográfica como el cuestionario aplicado a los expertos recolectaron una serie de instrumentos que se pueden utilizar para la gestión BIM hasta la quinta dimensión. Sin embargo, por consideración técnicas, económicas y de disponibilidad, muchas de las herramientas no se pueden implementar en el contexto ecuatoriano o no poseen retro compatibilidad entre ellas para desarrollar la gestión BIM propiamente dicha, es por esta razón que se eligieron las herramientas mostradas en la tabla 5.

Tabla 5. Instrumentos de gestión BIM a utilizar en el tramo Chiticay - Paute.

\begin{tabular}{ll}
\hline Dimensión & Herramienta \\
\hline BIM 2D & HydraFlow, plug-in 17utodesk-Subassambly Composer \\
BIM 3D & Civil CAD 3D \\
BIM 4D & Navisworks, Microsoft Project \\
\hline
\end{tabular}

Con las herramientas mencionadas en la tabla anterior se procedió a realizar el rediseño de la nueva sección del canal optimizado y se obtuvieron las cantidades de obra y presupuesto del proyecto, actividad que generalmente toma mucho tiempo a los ingenieros civiles. Con la finalidad de comparar los resultados entre la gestión tradicional y la gestión BIM se presenta en la tabla 6 y tabla 7 el presupuesto estimado para el tramo del canal Chiticay - Paute

Tabla 6. Presupuesto de construcción con gestión tradicional

\begin{tabular}{|c|c|c|c|c|c|c|}
\hline \multicolumn{7}{|c|}{ PRESUPUESTO } \\
\hline Ítem & Código & Descripción & Unidad & Cantidad & $\begin{array}{l}\text { P. } \\
\text { Unitario }\end{array}$ & P.total \\
\hline 1 & & Movimiento de tierras & & & & \\
\hline 1.1 & 502020 & Relleno & $\mathrm{m} 3$ & 2,90 & 18,24 & $\$ 52,90$ \\
\hline 2 & & Estructura & & & & \\
\hline 2.1 & 502001 & $\begin{array}{l}\text { Hormigón simple } \mathrm{f}^{\mathrm{c}} \mathrm{c}=180 \\
\mathrm{Kg} / \mathrm{cm} 2\end{array}$ & $\mathrm{~m} 3$ & 3,86 & 144,26 & $\$ 556,84$ \\
\hline & & Subtota & & & & $\$ 609,74$ \\
\hline & & IVA 12 & & & & $\$ 73,17$ \\
\hline & & Total por metr & ico & & & $\$ 682,91$ \\
\hline
\end{tabular}


Como se puede verificar en la tabla anterior las cantidades se calcularon por la metodología tradicional con las dimensiones tomadas en campo, con una sección promedio del canal existente, lo cual se restaría del volumen del canal para el relleno.

Tabla 7. Presupuesto de construcción con gestión BIM

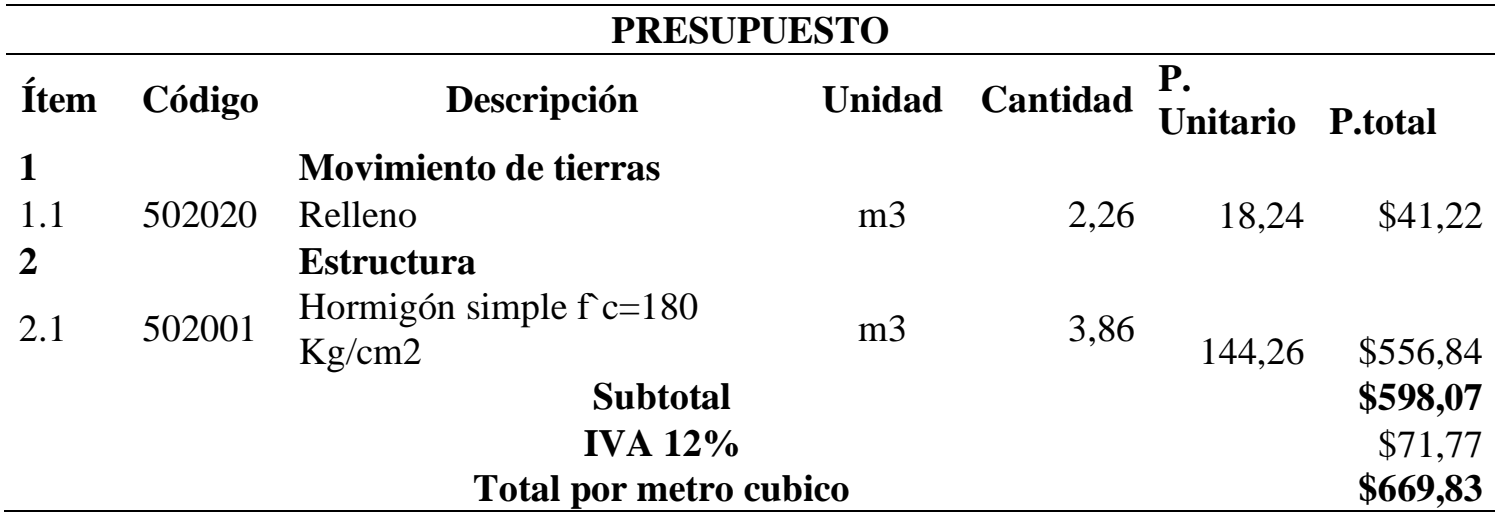

Se puede observar la variación que existe con la optimización de los recursos por medio de la metodología BIM en el tramo de canal, además se debe constatar que el sistema posee una longitud de $17 \mathrm{~km}$ lo cual sería eficiente para el mantenimiento.

El cálculo del hormigón del canal se mantiene debido a que las dimensiones son las mismas, pero en un imprevisto para el rediseño por medio de la metodología de la gestión BIM permite que la sección hidráulica varié (dinámicamente) para la generación de las nuevas cantidades de obra. En comparación de la metodología tradicional que se lo debería hacer repetitivamente. Además, se pudo verificar el sobredimensionamiento del canal antiguo con el canal nuevo obtenido por medio de la metodología de la gestión BIM.

\section{Conclusiones.}

- La integración de varias herramientas informáticas a través de la metodología BIM, dentro del campo de ingeniería civil, permite un ahorro notable de tiempo al calcular automáticamente: un óptimo diseño hidráulico de la sección, cantidad de materiales y presupuesto. A su vez, esta automatización de los procesos de gestión permite que las actividades del profesional afín también sean eficientes, pudiendo aprovechar el tiempo ahorrado en otras actividades relacionadas con la profesión.

- Luego de la revisión bibliográfica se puede observar el avance que tiene la metodología BIM en la proyección de nuevas construcciones a nivel mundial, por lo tanto, para que Ecuador se mantenga a la vanguardia en la gestión de proyectos y mejore los servicios prestados a la comunidad, debe incursionar y potenciar el uso de esta nueva tecnología. Además, puesto que Ecuador pertenece a la categoría de países en vías de desarrollo, debe invertir esfuerzos por optimizar las obras civiles y reducir el gasto público. En este sentido, al implementar esta metodología, reducirá considerablemente los costos por obra, aumentará ciclo de 
vida de los proyectos y permitirá a los consumidores aprovechar eficazmente el recurso hídrico sin interrupciones de subministro.

- En adición, se debe continuar explorando y adiestrando a los profesionales involucrados en obras civiles sobre la aplicación de la metodología BIM a otras etapas de la construcción de sistemas de riego como la distribución, e incluso operación del sistema, puesto que, como se demostró en esta investigación se puede lograr procesos eficientes y sostenibles en la gestión.

- Finalmente, pese a que en el desarrollo de esta investigación no se alcanza a desarrollar la quinta dimensión, la indagación bibliográfica y la encuesta a los expertos demuestra que se puede incorporar el factor costos a la gestión BIM, por lo tanto, se recomienda continuar ampliando esta investigación hasta complementar la 5D al proyecto.

- Agradecimientos: El presente artículo es parte del trabajo de investigación y titulación del Programa de Maestría en Construcción con Mención en Administración de la Construcción Sustentable de la Universidad Católica de Cuenca, por ello agradezco a todos y cada uno de los instructores por los conocimientos e información brindados para la elaboración del trabajo.

\section{Referencias bibliográficas.}

Banco Mundial. (22 de marzo de 2017). Grupo Banco Mundial. https://n9.cl/85jsy

Acampa, G. (2019). Representación del dibujo frente a simulación de los sistemas BIM. Oportunidad o amenaza para la arquitectura. ACE 40, 14(40), 111-132. doi: 10.5821/ace.14.40.6689

Bermejo, J., \& González, B. (2018). Aplicación de la metodología BIM al proyecto de construcción de un corredor de transporte para un complejo industrial -modelo BIM $4 d$ planificación [tesis de masterado, Escuela Técnica Superior de Ingeniería]. Repositorio de la ETSI. https://n9.cl/mi2mb

Cabré, R. (2012). Diseños cuasi-experimentales y longitudinales [tesis de grado, Universidad de Barcelona]. Repositorio de la UB. https://n9.cl/zjnl

Chow, V. (2004). Hidráulica de canales abiertos. 07-010776-9. McGraw-Hill. https://n9.cl/2qeq0

El Comercio. (15 de junio de 2014). Las juntas de agua marcan la vida de las comunidades en la Sierra. https://n9.cl/kgg3t

Gómez, P., Cubillo, F., \& Carrasco, F. (2017). Metodología para caracterizar la eficiencia de una red de distribución sectorizada. IMTA, 8(4), 57-77. https://n9.cl/6ec7

Goulven, Ribadeneira, \& Ruf. (1991). Riego tradicional andino en ecuador. Revista de desarrollo rural alternativo. (9), 177-198. https://n9.cl/uq34p

Montoya, Garrigos, Echarri, \& Rizo. (2020). Building Information Modelling: Disrupting Team Building and Teamwork on Construction Projects. Aplied Sciences, 1-18. 
Nieto, C., Pazmiño, E., Rosero, S., \& Quishpe, B. (2018). Estudio del aprovechamiento de agua de riego disponible por unidad de producción agropecuaria, con base en el requerimiento hídrico de cultivos y el área regada, en dos localidades de la Sierra ecuatoriana. Siembra, 5(1), 51-70. https://n9.cl/3e8rr

Olawumi, T., \& Chan, D. (2019). Building Information Modelling and Project Information Management Framework for Construction Projects. ResearchGate, 25(1), 53-75. DOI: 10.3846/jcem.2019.7841

Pérez, A., Leyva, D., \& Gómez, F. (2018). Desafíos y propuestas para lograr la seguridad alimentaria hacia el año 2050. Rev.Mex.Cienc.Agríc., 9(1). https://n9.cl/a625

Tang., Zhang., Guan. \& Chen. (2020). Integrating three-dimensional road design and pavement structure analysis. Automation in Construction, 2-14. https://n9.cl/hlafd

The United Nations World Water Development Report [WWDR]. (2019). Informe Mundial sobre el Desarrollo de los Recursos Hídricos 2015: Agua para un mundo sostenible. Water for a sustainable world. https://n9.cl/ylqp7

Vera, C. (2018). Aplicación de la metodología BIM a un proyecto de construcción de un corredor de transporte para un complejo industrial. Modelo BIM 5D Costes [tesis de fin de master, Universidad de Sevilla]. Repositorio de la US. https://n9.cl/rd9k

\section{LCiencia}




\section{PARA CITAR EL ARTÍCULO INDEXADO.}

Carlos Adrián, C. G., \& Coronel Sacoto, D. F. (2021). Aplicación de la metodología de la gestión BIM en el canal de conducción de agua del sistema de riego Chiticay - Paute. ConcienciaDigital, 4(3), 6-21. https://doi.org/10.33262/concienciadigital.v4i3.1761

\section{¿Ciencia}

El artículo que se publica es de exclusiva responsabilidad de los autores y no necesariamente reflejan el pensamiento de la Revista Conciencia Digital.

El artículo queda en propiedad de la revista y, por tanto, su publicación parcial y/o total en otro medio tiene que ser autorizado por el director de la Revista Conciencia Digital.

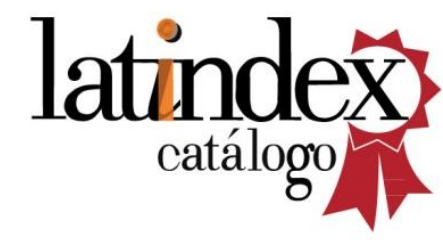

